\title{
MASE-EGTI - Simulador baseado em Teoria dos Jogos Evolucionários para Sustentabilidade Ambiental
}

\author{
Cássio Giorgio Couto Coelho ${ }^{1}$, Célia Ghedini Ralha ${ }^{2}$ \\ ${ }^{1}$ Departamento de Ciência da Computação - Instituto de Ciências Exatas \\ Universidade de Brasília \\ Campus Darcy Ribeiro - 70.910-900 - Distrito Federal - DF - Brasil \\ ${ }^{2}$ Departamento de Ciência da Computação - Instituto de Ciências Exatas \\ Universidade de Brasília \\ Campus Darcy Ribeiro - 70.910-900 - Distrito Federal - DF - Brasil \\ 160063035ealuno.unb.br, ghedini@cic.unb.br
}

\begin{abstract}
MASE, acronym for Multi-Agent System for Environmental Simulation, is a tool that was conceptualized for environmental simulations with anthropic exploration represented by BDI-cognitive agents. The current version is denominated MASE-BDI because of the cognition of their agents. MASE specializes in Land Use Cover Change models, with spatially-explicit agents exploring the natural environment according user-determined rules. This article introduces MASE-EGTI, a extension for MASE that presents social submodels based on Evolutionary Game Theory for spatial conflict resolution. The submodels not only represent social behavior, but also the environment exploratory profile of the agents. Equilibrium points of the strategies are elucidated in scenarios of physical space competition, and the simulation results are compared with the ones executed on MASE-BDI.
\end{abstract}

Resumo. MASE, uma ferramenta baseada em Sistemas Multiagentes para Simulação Ambiental, foi concebida para simulação em cenários de exploração em sistemas ambientais com agentes BDI-cognitivos. Por esse motivo, a versão mais atual da ferramenta é denominada MASE-BDI. MASE especializa-se em modelos de Land Use Cover Change, com agentes espacialmente posicionados explorando o meio natural de acordo com regras determinadas pelo usuário. Neste artigo apresenta-se o MASE-EGTI, que introduz submodelos sociais baseados em Teoria de Jogos Evolucionários para a resolução de conflitos de recursos espaciais pelos agentes do MASE. Tais submodelos não apenas refletem comportamentos sociais dos agentes, mas também perfis de exploratórios no ambiente. Pontos de equilíbrio das distribuições das estratégias são explorados em cenários de competição por espaço físico, e os resultados dos experimentos são comparados com os realizados no MASE-BDI.

\section{Introdução}

Sustentabilidade é um tema debatido em diversas disciplinas ligadas à Ecologia, e na última década tem tido especial atenção pela área de Ecologia de Paisagem [Opdam et al. 2018]. O interesse dessa área é tão importante que pesquisadores desse 
tópico cunharam o termo Landscape Sustainability Science (LSS), que procura reunir estudos a respeito de ambos conceitos em busca de soluções para questões relacionadas à resiliência e à longevidade de espaços naturais e antropizados [Wu 2013]. Uma vez que tanto mudanças climáticas quanto evoluções tecnológicas nos meios de produção são fatores que trazem transformações expressivas às paisagens, assim como diversos outros, os pesquisadores dessa nova abordagem utilizam técnicas e pesquisas na concepção de ações em busca do equilíbrio do uso de recursos naturais e do bem-estar humano.

O uso de modelos espacialmente explícitos, simulação computacional e verificação e validação são essenciais em estudos em LSS [Wu 2013]. Diversas aplicações e frameworks têm sido usados ao longo dos anos em estudos em Land Use Cover Change (LUCC), subárea de Ecologia de Paisagens e consequentemente área correlata a LSS. IDRISI, ArcGIS, DinamicaEGO e MASE são algumas dessas ferramentas computacionais com várias abordagens e métodos, utilizadas em casos de usos em LUCC com bons resultados [Coelho et al. 2016]. Apesar dessa proximidade entre Computação e Ecologia de Paisagens e dessa produção de modelos e resultados importantes, ainda é grande desafio de ambas áreas o aperfeiçoamento de ferramentas de simulação que auxiliem o encontro de pontos de equilíbrio entre preservação ambiental e bem-estar humano, além de representar fenômenos de uso e transformação do ambiente de forma satisfatória. Nesse sentido a questão de pesquisa que este artigo apresenta é: uma ferramenta de simulação baseada em agentes com Teoria dos Jogos Evolucionários (TJE) pode evidenciar os pontos de equilíbro do uso sustentável de recursos naturais com bem-estar social?

Este artigo apresenta o Mase-Agent System for Environmental Simulation with Evolutionary Game Theory Interactions (MASE-EGTI), extensão do simulador MASE na versão MASE-BDI [Coelho et al. 2016] com a introdução de modelos em TJE para a caracterização dos agentes transformadores em suas interações sociais. A Seção 2 mostra conceitos e estruturas utilizadas para a concepção do MASE-EGTI, e a Seção 3 descreve essa extensão brevemente, bem como sua nova arquitetura. A Seção 4 ilustra o estudo de caso utilizado e mostra as variações na distribuição da população de agentes e consequentes interações entre eles, bem como uma breve comparação com os resultados obtidos no MASE-BDI. Por fim, a Seção 5 apresenta um fechamento deste artigo, com os próximos passos dessa pesquisa.

\section{Materiais e Métodos}

Duas premissas fundamentais dos modelos executados no MASE são: a discretização do espaço em células e a ocupação de somente um agente por célula por intervalo de tempo. Isso pode levar a competição de espaço entre agentes interessados no mesmo recurso espacial em um mesmo intervalo de tempo. A resolução desses conflitos é uma motivação inicial para a introdução de modelos sociais baseados em TJE para a decisão de qual agente deve obter o bem almejado. Além disso, a associação de um perfil comportamental pode não apenas modelar uma estratégia em um jogo de TJE, mas configurar a agressividade da exploração dos recursos ambientais por parte do agente. Sendo assim, os conflitos espaciais não apenas determinam o sucesso de uma estratégia social sobre a outra, mas também influenciam o processo de transformação da paisagem como consequência. A inclusão de políticas públicas no modelo estudado pode então adequar a distribuição de comportamentos agressivos e pacíficos na população de agentes, com o objetivo do encontro de um ponto de equilíbrio entre exploração do espaço e preservação 
do meio ambiente.

\subsection{Landscape Sustainability Science}

LSS é uma área de estudo recente, com a criação do termo em um artigo publicado em 2013 [Wu 2013]. No entanto, suas motivações e objetos de estudo já possuiam raízes em áreas correlatas há mais tempo, em áreas que, apesar de próximas, eram associadas de formas distintas: Sustentabilidade e Ecologia de Paisagens. [Potschin and Haines-Young 2006] em seu artigo "'Rio+10', sustainability science and Landscape Ecology" fazem uma reflexão sobre a necessidade de Sustentabilidade e Ecologia de Paisagens serem tratadas de uma forma mais interdisciplinar, motivados por discussões técnicas presenciadas nas conferências ECO-92 (também conhecida como Earth Summit ou Rio Summit 92), em 1992, e a Rio+10, em 2002, em Joanesburgo. Nesse período de 10 anos, o volume de pesquisa em ambas áreas levaram a membros da comunidade científica a entender que ambos assuntos possuem uma grande quantidade de pontos de apoio mútuo.

Nesse mesmo artigo, [Potschin and Haines-Young 2006] propõem o "Modelo da Língua" (Tongue Model, Figura 1), batizado por causa do seu formato característico quando representado graficamente. Esse modelo conceitual utiliza temas debatidos em ambas áreas, ilustrando didaticamente que o equilíbrio entre interesses antropocêntricos e a capacidade do meio ambiente em fornecer recursos de forma sustentável não é entendido como um único ponto, mas sim como uma área ótima em que ambas características se encontram - tanto a necessidade humana por bens e serviços quanto a capacidade do meio ambiente em entregar recursos sem comprometer cenários futuros de sustento próprio e da sociedade. Tais características são entendidas como interdependentes, e no caso da capacidade de geração de recursos do meio-ambiente, potencialmente finita.

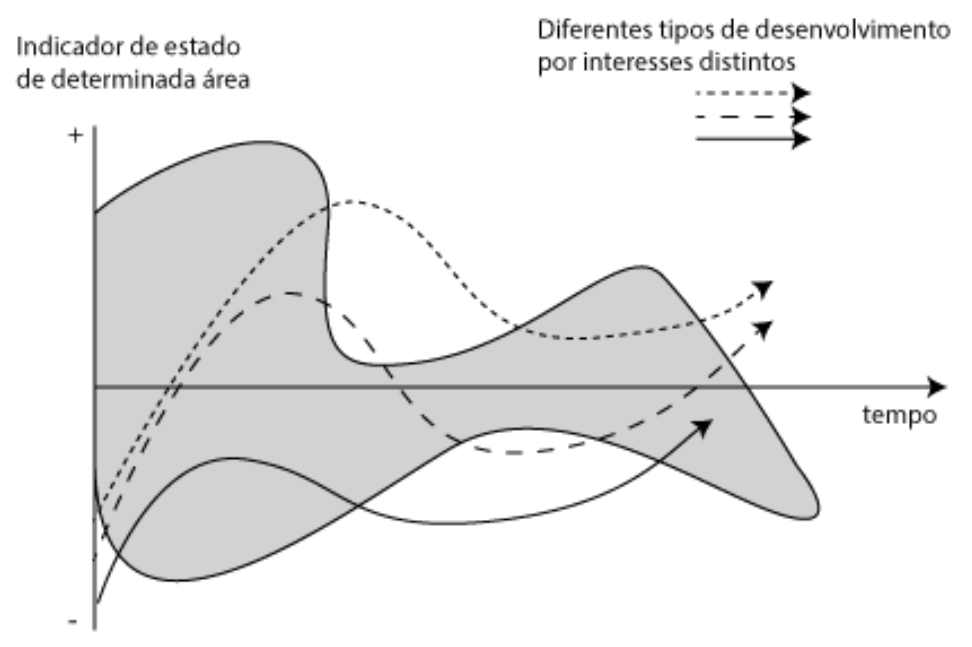

Figura 1. 0 modelo da língua. Adaptado e traduzido de [Potschin and Haines-Young 2006]

\subsection{Teoria dos Jogos Evolucionários}

TJE é área derivada de Teoria dos Jogos (TJ), de Economia, e estuda situações estratégicas em que agentes pertinentes a uma população exibem comportamentos variados em suas 
interações [Smith 1988]. Também é alvo do estudo dessa área o porquê da ocorrência de um comportamento predominante em determinados casos, e se esse comportamento é estável - ou seja, se mantém majoritário mesmo com a pertubação do equilíbrio pela mutação ou imigração de indíviduos de comportamentos divergentes.

Os jogos em TJE possuem duas características que os diferenciam dos jogos clássicos de Teoria dos Jogos. Eles são situações estratégicas que ocorrem múltiplas vezes em sequência, como uma representação dos indíviduos interagindo continuamente em sociedade, diferentemente de TJ, que normalmente estuda a situação em si sem ter preocupações com próximas interações continuamente. Além disso, os agentes jogadores não necessariamente escolhem a melhor estratégia racionalmente em TJE, ao contrário de TJ, em que eles necessariamente procuram a estratégia mais lucrativa ou menos punitiva. As estratégias dos agentes em TJE são imaginadas uma manifestação de um fenótipo, de um genótipo, de um comportamento ou de uma característica apresentada que reflete na sobrevivência e evolução de um organismo no meio [Weibull 1997].

\subsubsection{Perfis Estratégicos e Jogos Evolucionários}

Os perfis estratégicos são caracterizações de todos os comportamentos observáveis na população de agentes, apresentados como o conjunto $S=\left\{S_{0}, S_{1}, S_{2} \ldots S_{\mathrm{n}}\right\}$. Um jogo evolucionário é uma situação estratégica onde um ou mais agentes interagem de acordo com seus respectivos comportamentos, obtendo um resultado denominado payoff. $\mathrm{O}$ payoff é a mensuração da recompensa ou punição da interação de um agente de comportamento $\alpha$ com outro de comportamento $\beta$, e reflete a adaptabilidade (fitness) de indíviduo. Dessa forma, um jogo é normalmente representado pela matriz de payoff, que possui todos as possibilidades de encontro entre dois jogadores com perfis estratégicos próprios e suas possíveis consequências. A Tabela 1 demonstra as estratégias e payoffs do jogo Hawk-Dove, baseado no dilema do prisioneiro, clássico de Teoria de Jogos. Nesse exemplo, o conjunto $S$ de perfis estratégicos é totalmente descrito por $S=\{$ Hawk, Dove $\}$. Os valores $V$ e $C$ representam o valor de um recurso desejável e o custo de um embate físico, respectivamente.

Nesse jogo, dois agentes almejam um mesmo recurso de valor $V$, e ambos possuem perfis estratégicos próprios. Se ambos agentes forem Hawks, ambos lutam pelo recurso $V$ e acabam dividindo-o - porém, acabam por dividir o custo $C$ por terem entrado em um embate. Se ambos forem Doves, eles dividem o recurso $V$ harmonicamente, sem lutas. Se um agente for Hawk e outro for Dove, o primeiro toma o recurso por completo, enquanto o segundo recebe uma penalidade de custo $C$, como se tivesse sido coagido a ceder o recurso de forma violenta.

Outro jogo bastante conhecido em TJE é o Rock-Paper-Scissors, em que há três perfis estratégicos: $S=\{$ Rock, Paper, Scissors $\}$. Tal como o conhecido jogo de "pedra, papel e tesoura", cada estratégia é forte contra uma, fraca contra outra e inefetiva contra si mesma. 
Tabela 1. O Jogo Hawk-Dove, proposto por Maynard e Price em 1973 [Weibull 1997].

\begin{tabular}{|c|c|c|c|}
\hline & \multicolumn{2}{|c|}{ Jogador 2} \\
\hline & & Hawk & Dove \\
\hline \multirow[t]{2}{*}{ Jogador 1} & Hawk & $\left(\frac{V-C}{2}, \frac{V-C}{2}\right)$ & $(V,-C)$ \\
\hline & Dove & $(-C, V)$ & $\left(\frac{V}{2}, \frac{V}{2}\right)$ \\
\hline
\end{tabular}

\subsubsection{Estratégias Evolucionárias Estáveis}

De uma forma geral, um comportamento é considerado uma Estratégia Evolucionária Estável (Evolutionary Stable Strategy, ESS) se sua predominância na população não é perturbada por outros comportamentos possíveis na população. Sob outra perspectiva, uma ESS $\theta$ possui payoffs superiores ao de outras estratégias possíveis, e por isso reflete maior adaptabilidade de indíviduos que apresentam comportamento $\theta$ que outros.

Sendo assim, considera-se conjunto de perfis de estratégia $P=\{S, T\}$, e quatro indíviduos, $I_{S 1}$ e $I_{S 2}$ que apresentam comportamento $S$, e $J_{T 1}$ e $J_{T 2}$ que apresentam comportamento $T$. Pode-se considerar $S$ uma ESS se uma das condições é satisfeita:

- O payoff de um indíviduo $I_{S 1}$ que encontra o indíviduo $I_{S 2}$ deve ser maior que o payoff de $I_{S 1}$ quando encontra o indíviduo $J_{T 1}$;

- O payoff de um indíviduo $I_{S 1}$ que encontra o indíviduo $I_{S 2}$ é igual ao payoff de $I_{S 1}$ quando encontra o indíviduo $J_{T 1}$. No entanto, o payoff de um indíviduo $I_{S 1}$ que encontra o indíviduo $I_{T 1}$ é maior que payoff de $J_{T 1}$ quando encontra o indíviduo $J_{T 2}$.

\section{MASE-EGTI}

A introdução de modelos de interação baseados em TJE no MASE-BDI busca explorar simulações em modelos de LUCC que almejam o alcance da sustentabilidade de recursos e bem-estar social no meio ambiente. Agentes apresentando diferentes comportamentos podem expressar indíviduos mais ou menos adaptados à cenários de equilíbrio, sustentação e preservação de recursos espaciais. Políticas públicas, que incentivam ou inibem a ação de agentes no espaço, podem causar uma mudança na adaptabilidade dos comportamentos. Por exemplo, políticas de ordenamento do solo (PDOT) são comumente exploradas nos estudos de caso do MASE independentemente de sua versão [Coelho et al. 2016].

Sendo assim, MASE-EGTI foi construído como extensão da ferramenta MASEBDI com algumas mudanças em sua arquitetura interna com a utilização de linguagem Java 8 e o framework JADEX [Braubach and Pokahr 2012] na versão 2.5. O MASEBDI simula modelos que são descritos por imagens dos espaços a serem explorados, por imagens de seus atributos espaciais e por regras estruturadas de movimentação e ocupação. Além disso, políticas públicas podem ser inseridas para a ordenação da exploração dos agentes. O MASE-EGTI herda esse funcionamento básico e acrescenta novas configurações a respeito de perfis estratégicos dos agentes, de jogos representativos das situações de conflito e de distribuição de perfis na população implementada na 
simulação, e busca por meio de experimentações evidenciar possíveis ESS e suas consequências na extração de recursos naturais do solo.

\subsection{Arquitetura}

Quanto à arquitetura, [Coelho et al. 2016] descreve o MASE-BDI como uma aplicação de três camadas principais - Interface com o usuário, Utilidades e Agentes -, e com agentes hierarquicamente divididos em gerentes e agentes transformadores. Há um gerente que administra a simulação em si (GRID Manager, GM), um que administra os recursos espaciais (Spatial Manager, SM), um que administra os agentes transformadores (Transformation Manager, TM) e um tipo genérico, que pode administrar outros recursos (Other Managers). Os agentes transformadores (AT) são fisicamente representados em um grid de simulação, e ocupam e transformam os espaços de acordo com regras determinadas pelo usuário da aplicação. Todos os agentes possuem um modelo cognitivo baseado em Belief-Desires-Intentions [Rao and Georgeff 1995].

No MASE-EGTI as camadas do software se mantém , porém a arquitetura dos agentes é mais condensada (Figura 2). O Gerente de Simulação (GSIM) passa a herdar as tarefas dos GM e SM da arquitetura anterior. O TM e os gerentes de tipos genéricos são extintos. Os AT se mantém, dessa vez agrupados pelos seus perfis estratégicos prédefinidos pelo usuário, e passam a migrar de grupo de acordo com suas deliberações internas a respeito do próximo perfil adotado ao longo da simulação.

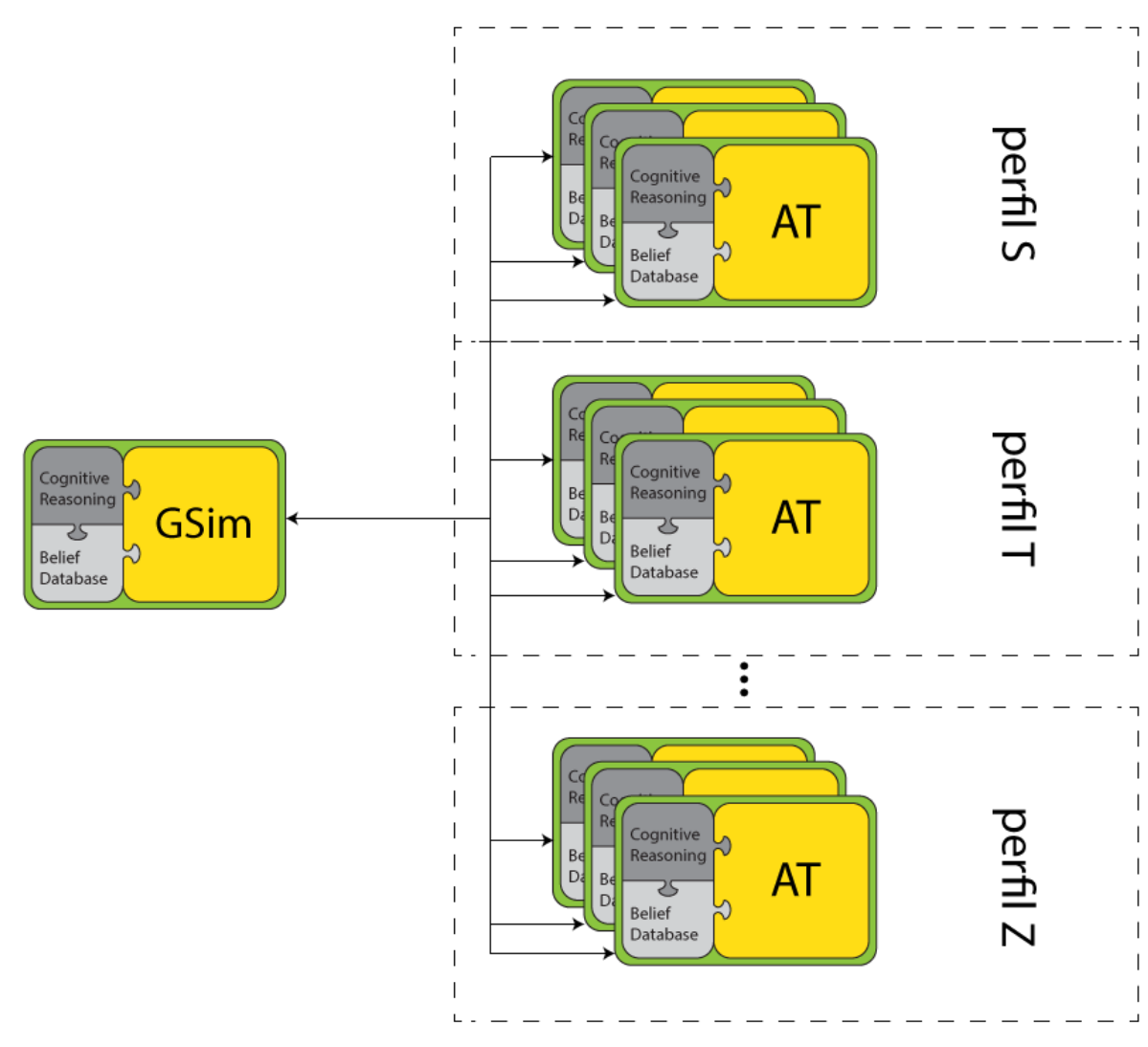

Figura 2. A arquitetura dos agentes do MASE-EGTI 


\subsection{Interações entre agentes}

Similar ao funcionamento do MASE-BDI, o MASE-EGTI possui steps - passos de simulação em que os agentes executam um conjunto de ações baseado em seus comportamentos registrados e o potencial de recursos que o espaço tem a oferecer. Uma vez que o potencial de recursos de uma célula zera, o agente move-se para uma célula de sua vizinhança. Em uma simulação com uma quantidade de agentes suficiente, conflitos de espaço podem ocorrer, uma vez que dois agentes não podem ocupar o mesmo espaço em um mesmo step. Nessas situações, os jogos evolucionários e as políticas públicas entram em ação para que os agentes decidam entre si qual deles irá conquistar o recurso espacial.

Considerando o jogo Hawk-Dove como exemplo (Tabela 1), temos a situação em que um agente Hawk encontra um agente Dove. Nesse caso, o ganhador é o agente Hawk, que conquista o recurso espacial, enquanto o agente Dove é obrigado a escolher outra célula. No entanto, em caso de empate, o ganhador é decidido aleatoriamente por meio de uma aposta - o agente que tirar o maior número de um gerador aleatório ganha o espaço. Os payoffs recebidos são utilizados para um registro interno dos agentes de sucesso de uma estratégia. Cada perfil estratégico é registrado com o somatório dos payoffs recebidos a cada interação - sejam positivos, negativos ou neutros. Uma vez que um agente perde uma célula em uma interação, ele pode ponderar trocar de estratégia, ou seja, consultar seus registros e ver que estratégia é mais vantajosa de acordo com suas experiências passadas. Esse mecanismo de troca é denominada "melhor payoff acumulado" no MASE-EGTI.

Alternativamente, o usuário do MASE-EGTI pode configurar os agentes perdedores para trocar de estratégia em um mecanismo de "vingança". O agente que perde um conflito pode simplesmente trocar para a estratégia que teria chance de ganhar do embate que acabou de perder. Por exemplo, em um jogo de Rock-Paper-Scissors, um agente "Rock" que perdera um espaço para um agente "Paper" poderia realizar a troca de estratégia para "Scissor" sob essa configuração de mecanismo.

\section{Estudo de Caso}

Para a ilustração do funcionamento do MASE-EGTI, escolheu-se o estudo de caso Cerrado-DF, descrito em [Coelho et al. 2016] (Figura 3). Este é um modelo bastante representativo por ilustrar o uso e a transformação de solo no Distrito Federal entre os anos 2002 e 2008 (Figura 4). Essa unidade federativa é interessante por possuir um único bioma em toda sua extensão - o Cerrado, o segundo maior bioma brasileiro, ameaçado nos últimos anos pela rápida expansão agrícola, pecuária e urbana [Sano et al. 2008]. $\mathrm{O}$ modelo Cerrado-DF considera agentes transformadores pertinentes à duas atividades sócio-econômicas principais: pecuária e agricultura. Ele também leva em consideração a representação de grandes companhias produtoras (agentes "de grupo") e produtores locais, principalmente de subsistência (agentes "individuais"). A essa característica associou-se um perfil estratégico pertencente ao jogo Hawk-Dove, em que os valores de $V$ e $C$ da Tabela 1 assumem, respectivamente, 1 e -2 (Tabela 2). Considerando na Figura 4 que os espaços amarelos possuem 500 pontos de potencial de entrega de recursos e os verdes, 1500, a Tabela 3 mostra os agentes caracterizados em termos de exploração e perfil estratégico.

Para a execução da simulação do Cerrado-DF no MASE-EGTI foram confi- 
Tabela 2. O Jogo Hawk-Dove com os payoffs utilizados na simulação.

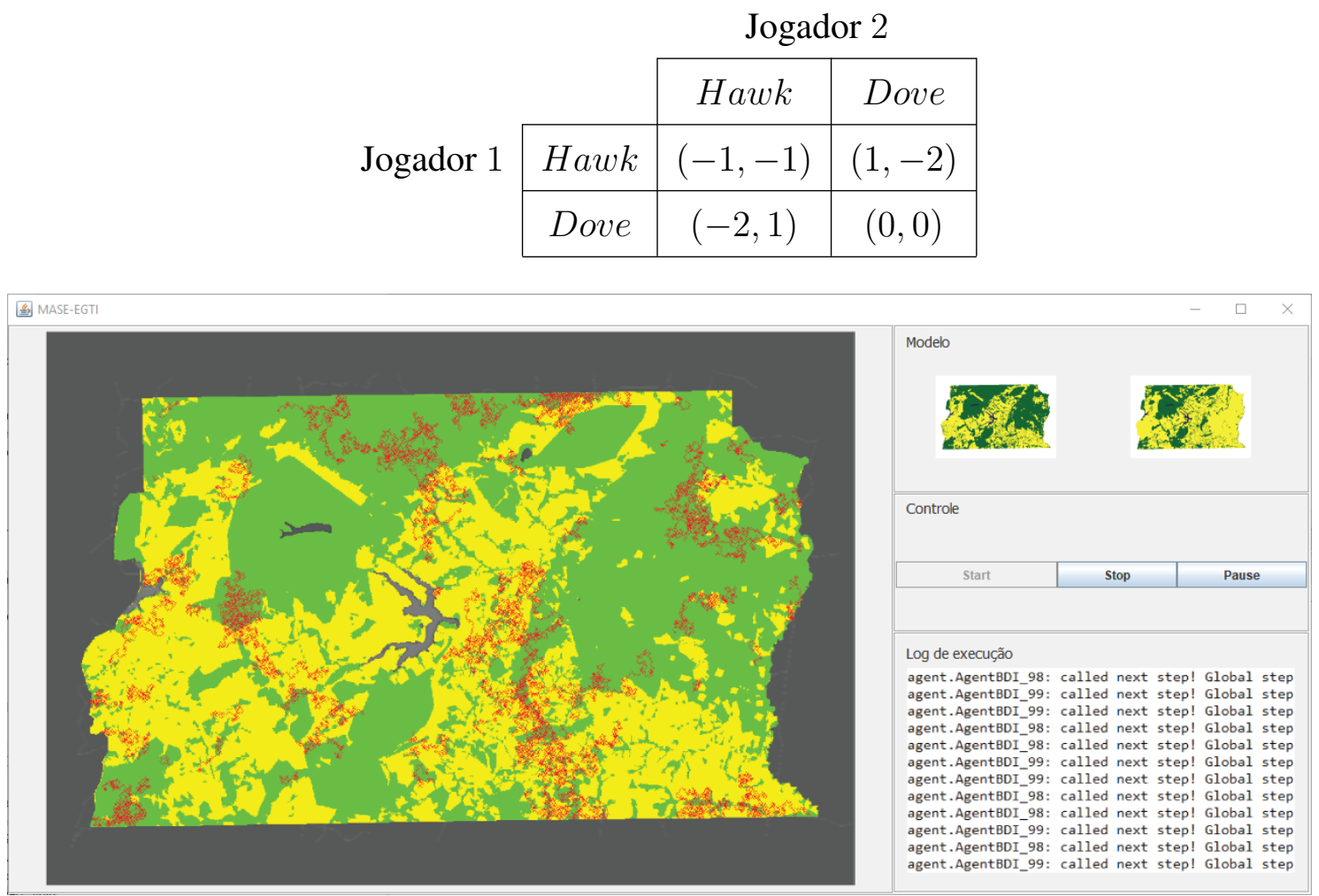

Figura 3. A execução do modelo Cerrado-DF no MASE-EGTI.

gurados 100 agentes aleatoriamente pelo GRID de simulação, com distribuição $P=$ $\{$ Hawk $=0.5$, Dove $=0.5\}$ e 365 steps de simulação. Nesse cenário, foram feitas duas execuções: uma com o mecanismo de troca de estratégia "vingança"e outra com "melhor payoff acumulado". A cada 10 steps, a quantidade de agentes por estratégia foi aferida, e a evolução da distribuição inicial foi registrada nos gráficos apresentados da Figura 5.

Em ambos casos, o equilíbrio populacional é atingido. No caso do mecanismo de "vingança" o equilíbrio é trivial - uma vez que a a única estratégia capaz de ter possibilidade de ganho uma sobre a outra ser Hawk, rapidamente todos os agentes migram e permanecem nela. No entanto, no caso do mecanismo "melhor payoff acumulado" o equilíbrio é melhor distribuído na população. Isso porque Hawk é uma estratégia mais lucrativa que Dove. Hawk é uma estratégia atende a primeira condição apresentada na Seção 2.2.2, o que o caracteriza com ESS. No entanto, o acúmulo excessivo de Hawks nesse caso poderia ser prejudicial ao lucro acumulado percebido - fazendo alguns membros a voltarem a ser Dove reequilibrando a distribuição.

Após essa análise, executou-se 10 experimentos com a quantidade de agentes iniciando em 10 e acrescentando-se 10 a cada execução. Em todas as execuções a distribuição inicial de comportamentos foi $P=\{H a w k=0.5$, Dove $=0.5\}$ e com mecanismo "melhor payoff acumulado". O propósito dessas execuções foi para o cálculo da Figura de Mérito (FoM), métrica estabelecida por [Pontius et al. 2008] e utilizada em [Coelho et al. 2016] para a aferição do modelo em termos objetivos. Comparativamente, 


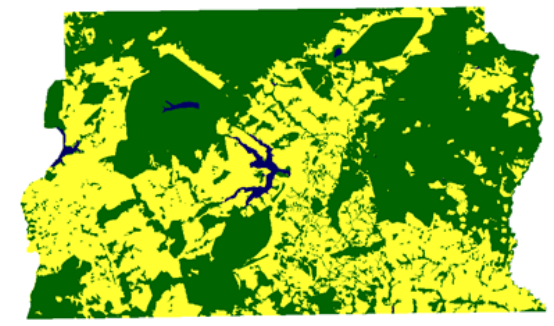

2002

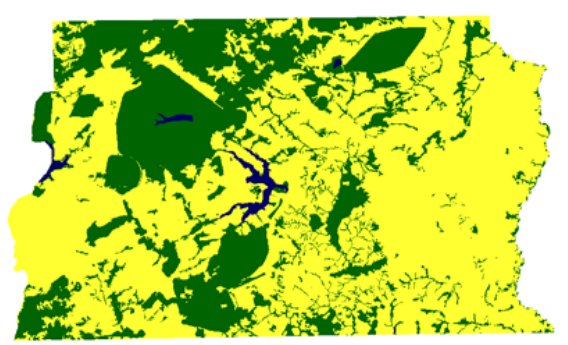

2008

Figura 4. Representações gráficas dos anos de 2002 e 2008 . Os espaços amarelos representam áreas antropizadas, enquanto os verdes representam mata nativa.

Tabela 3. A associação das capacidades de exploração e dos perfis estratégicos dos agentes transformadores do MASE-EGTI

\begin{tabular}{|l|c|c|}
\hline & Potencial de exploração/step & Perfil estratégico \\
\hline Agente de Grupo & 1500 & Hawk \\
\hline Agente Individual & 500 & Dove \\
\hline
\end{tabular}

enquanto MASE-BDI obteve os melhores FoM entre 50\% e 55\%, MASE-EGTI possuiu métricas mais baixas, variando entre $33 \%$ e $37 \%$ com o mesmo modelo, porém com as novas estruturas baseadas em TJE.

\section{Conclusão}

Neste artigo foi apresentada a ferramenta MASE-EGTI, extensão da MASE-BDI com a introdução de TJE para a resolução de conflitos espaciais entre agentes transformadores do espaço e para a caracterização dos perfis de comportamento e exploração em modelos em LUCC. Enquanto o bem-estar social é consequência da produção de recursos extraídos do meio ambiente, o volume e a velocidade da extração de recursos naturais em determinadas áreas é fator determinante para a sobrevivência e longevidade delas, conforme evidencia [Potschin and Haines-Young 2006] no "Modelo da Língua", ilustrado na Figura 1. Sendo assim, identificar que tipo de comportamento extrativista é mais atuante na sociedade é fundamental para o desenvolvimento de políticas públicas que controlem o impacto sobre áreas de desenvolvimento sustentável.

A dominância de um modo de exploração agressivo é sugerido pelos primeiros experimentos realizados no MASE-EGTI com o modelo Cerrado-DF. Em ambos cenários, o equilíbrio de comportamentos indicou a predominância de extrativismo predatório, com o objetivo do aumento de lucros e aumento de produção para a sociedade. Sendo assim, é preciso desenvolver novos experimentos com a introdução de políticas públicas que incentivam o extrativismo sustentável e assim encontrar novas áreas de equilíbrio entre interesse público e sustentabilidade ambiental, e assim evidenciar modelos em que o desenvolvimento sustentável é possível. 

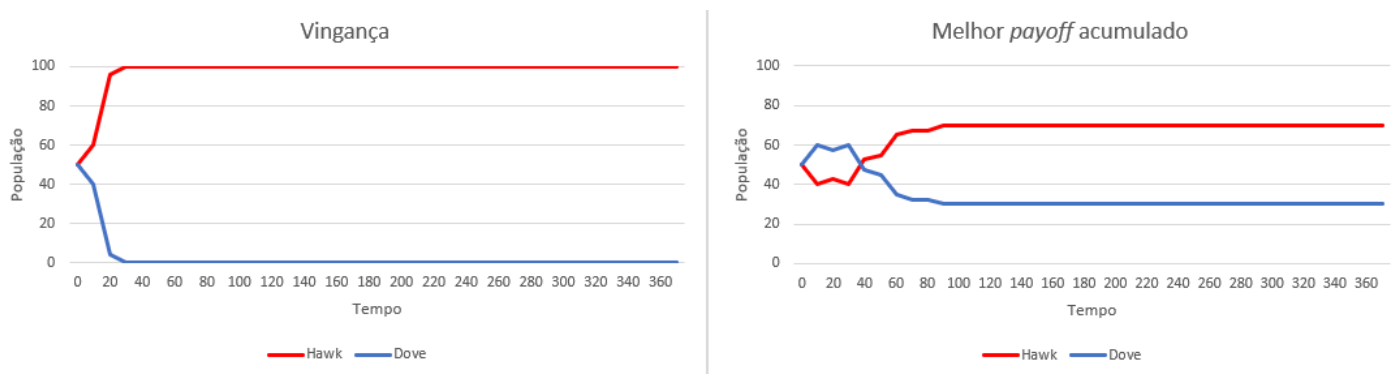

Figura 5. Gráficos da distribuição de comportamentos na população ao longo da simulação com mecanismos de troca distintos.

\section{Referências}

Braubach, L. and Pokahr, A. (2012). Jadex active components framework-bdi agents for disaster rescue coordination. Software agents, agent systems and their applications, 32:57-84.

Coelho, C. G. C., Abreu, C. G., Ramos, R. M., D. Mendes, A. H., Teodoro, G., and Ralha, C. G. (2016). Mase-bdi: agent-based simulator for environmental land change with efficient and parallel auto-tuning. Applied Intelligence, 45(3):904-922.

Opdam, P., Luque, S., Nassauer, J., Verburg, P. H., and Wu, J. (2018). How can landscape ecology contribute to sustainability science? Landscape Ecology, 33(1):1-7.

Pontius, R. G., Boersma, W., Castella, J.-C., Clarke, K., de Nijs, T., Dietzel, C., Duan, Z., Fotsing, E., Goldstein, N., Kok, K., Koomen, E., Lippitt, C. D., McConnell, W., Mohd Sood, A., Pijanowski, B., Pithadia, S., Sweeney, S., Trung, T. N., Veldkamp, A. T., and Verburg, P. H. (2008). Comparing the input, output, and validation maps for several models of land change. The Annals of Regional Science, 42(1):11-37.

Potschin, M. and Haines-Young, R. (2006). rio+10, sustainability science and landscape ecology. Landscape and Urban Planning, 75(3):162 - 174. Landscapes and sustainability.

Rao, A. S. and Georgeff, M. P. (1995). Bdi agents: From theory to practice. In Proceedins of the First International Conference on Multi-Agent Systems (ICMAS-95), pages 312319.

Sano, E. E., Rosa, R., Brito, J. L. S., and Ferreira, L. G. (2008). Mapeamento semidetalhado do uso da terra do Bioma Cerrado. Pesquisa Agropecuária Brasileira, 43(1):153-156.

Smith, J. M. (1988). Evolution and the Theory of Games, pages 202-215. Springer US, Boston, MA.

Weibull, J. W. (1997). Evolutionary game theory. MIT press.

$\mathrm{Wu}$, J. (2013). Landscape sustainability science: ecosystem services and human wellbeing in changing landscapes. Landscape Ecology, 28(6):999-1023. 